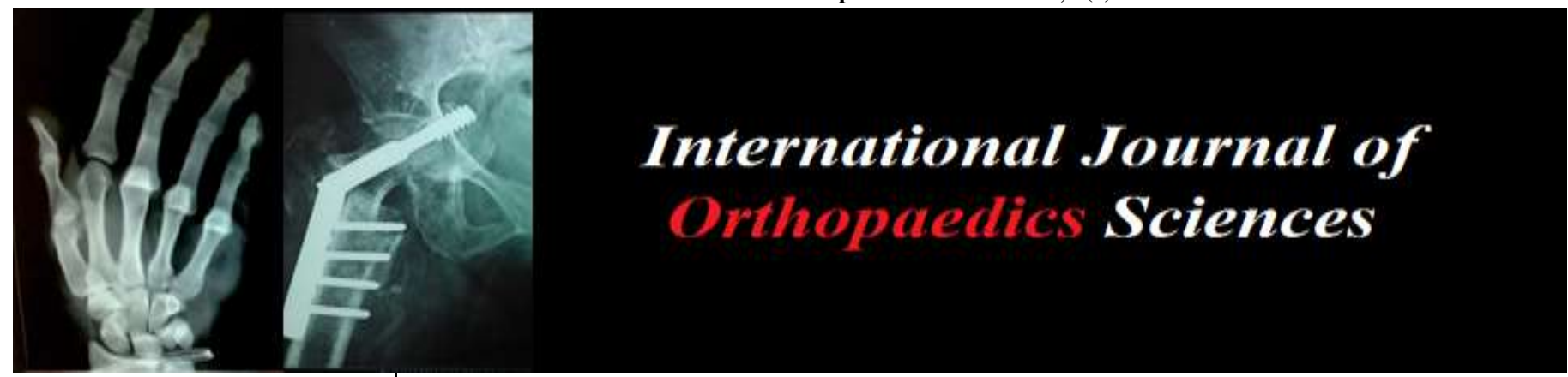

E-ISSN: 2395-1958

P-ISSN: 2706-6630

IJOS 2020; 6(4): 759-764

(C) 2020 IJOS

www.orthopaper.com

Received: 12-09-2020

Accepted: 21-10-2020

Dr. Mohammed Assad Hasan M.B. Ch.B., Iraq/Medical City Complex/Ghazi All-Hariry Hospital, Iraq

Dr. Mahmmood Shihab Wahab M.B. Ch. B., F.I.C.M.S Iraq/ Medical City Complex/Ghazi AllHariry Hospital, Iraq

Dr. Ali Fawzi Khiraldeen M.B. Ch. B., F.I.C.M.S Iraq/ Medical City Complex/Ghazi AllHariry Hospital, Iraq
Corresponding Author: Dr. Mohammed Assad Hasan M.B. Ch.B., Iraq/ Medical City Complex/Ghazi All-Hariry Hospital, Iraq

\section{The efficacy of local infiltrative analgesia in total knee arthroplasty regarding pain and movement in early postoperative period}

\section{Dr. Mohammed Assad Hasan, Dr. Mahmmood Shihab Wahab and Dr. Ali Fawzi Khiraldeen}

DOI: $\underline{\text { https://doi.org/10.22271/ortho.2020.v6.i4k.2417 }}$

\section{Abstract}

Background: Total knee arthroplasty is a successful procedure for relieving pain and restoring function in cases with severe rheumatoid arthritis and osteoarthritis. Local infiltration analgesia is becoming more commonly used owing to the excellent pain relief, the low frequency of complications, and the antiinflammatory effect.

Objective: To evaluate the efficacy of local infiltrative analgesia when it's uses in total knee arthroplasty patients for decrease the pain score and increase range of motion in early post-operative rehabilitation. Methods: a comparative prospective study carried in the period from October 2016 to September 2018, was applied for 36 patients in Medical City, Hospital of Specialized Surgeries and Private Nursing Home Hospital.

Results: In group A, means of ROM score one day and two days postoperatively were decreased than that before operation ( 105.12 versus $89.37, \mathrm{P}=0.001$ and 105.12 versus $87.37, \mathrm{P}=0.001$ respectively). Ingroup $\mathrm{B}$, means score one day and two days postoperatively were decreased than that before operation (105.75 versus $88.50, \mathrm{P}=0.001$ and 105.75 versus $82.75, \mathrm{P}=0.001$ respectively). During rest, means of VAS score four hours, 12 hours, one day, and two days after operation were significantly decreased in (group A) compared to group B. During movement, insignificant differences in means of VAS score between study groups four hours, 12 hours, and two days after operation $(\mathrm{P}=0.258,0.057$, and 0.284 respectively).

Conclusion: Local infiltration analgesia effectively reduce pain scores during rest and movement also reduces hospital stay in patients undergoing total knee arthroplasty, and better physiotherapy outcome.

Keywords: Total knee arthroplasty, ROM score, VAS score, local infiltration analgesia

\section{Introduction}

Anatomy of knee joint

The knee joint is the largest and one of the most complex joints in the human body. A unique interaction of bones, muscles, menisci, and ligaments results in a compromise between stability and mobility ${ }^{[1]}$. Basically, it consists of two condylar joints between the medial and lateral condyles of the femur and the corresponding condyles of the tibia, and a gliding joint, between the patella and the patellar surface of the femur. Above are the rounded condyles of the femur; below are the condyles of the tibia and their cartilaginous menisci; in front is the articulation between the lower end of the femur and the patella. The articular surfaces of the femur, tibia, and patella are covered with hyaline cartilage ${ }^{[2]}$.

\section{Innervation of the knee joint}

The neural innervation of the knee joint is complex which innervated by the articular branches of the muscles which move the joint. The articular nerves are derived from the femoral, obturator, tibial, common peroneal, and recurrent peroneal nerves. The femoral nerve, through its saphenous branch and also via its branches to the vast us medial is, intermedium, and lateralis muscles, supplies the suprapatellar recess, the patellar periosteum, and the anteromedial and anterolateral portions of the joint capsule. The medial, lateral, and posterior aspects of the joint capsule, the infrapatellar fat pad, tibial periosteum, and the superior tibio- 
fibular joint are supplied by the tibial nerve. The common peroneal nerve supplies the anterolateral portion of the capsule, the infrapatellar fat pad, and the tibial periosteum. The obturator nerve supplies the superior part of the posteromedial capsule and the anteromedial aspect of the capsule. Cutaneous innervation of the anterior aspect of the knee is supplied by the femoral nerve ${ }^{[3]}$.

Total Knee Arthroplasty (TKA) ${ }^{[4]}$.

Total knee arthroplasty (TKA) is the surgical treatment of choice for advanced osteoarthritis after failure of other modes of conservative management in properly selected patients. There are two designs of implants used for TKA:

-Unconstrained: which include cruciate retaining, Cruciate substituting and mobile bearing knees.

-Constrained: which include hinged and non-hinged. However unconstrained type is the most common type, used for uncomplicated knee problems, artificial components inserted into the knee are not linked to each other, have no stability built into the system, and relies on the patient's own ligaments and muscles.

\section{Goal of TKA}

1. Pain relief.

2. Restoration of normal limb alignment.

3. Restoration of a functional range of motion.

\section{Technical Goals of TKA}

1. The restoration of mechanical alignment

2. Preservation (or restoration) of the joint line.

3. Balanced ligaments.

4. Maintaining or restoring a normal Q angle.
Absolute contraindications for TKA
1. Recent or current knee sepsis.
2. Remote source of ongoing infection
3. Extensor mechanism discontinuity or sever dysfunction.
4. Recurvatum deformity secondary to muscular weakness.
5. Presence of painless well functioned knee arthrodesis

\section{Post-operative analgesia}

Postoperative Total knee arthroplasty (TKA) analgesia remains a challenging issue. It is reported that more than half of the patients undergoing knee replacement would experience severe pain in the early postoperative period Considerable postoperative pain interferes with patients' participation in physiotherapy, prolongs inpatient stay, lowers patient satisfaction, and leads to chronic pain and dysfunction. Thus, effective pain control in the immediate postoperative period is crucial for patients' convalescence after TKA ${ }^{[5]}$.

Local infiltration analgesia (LIA) is becoming more commonly used owing to the excellent pain relief, the low frequency of complications, and the anti-inflammatory effect [6]. Options for postoperative pain control include patient administered narcotics, epidural anesthetics, and spinal anesthetics with adjuncts such as long-acting morphine and peripheral nerve blocks (with and without catheters). These concepts are widely used, but there are reports of multiple side effects secondary to parenteral opioids and problems associated with motor blockade after nerve blocks, which can lead to delays in rehabilitation ${ }^{[7]}$.

Local infiltration analgesia is simple, practical, safe, and effective for pain management after knee surgery. We describe a multimodal technique for control of acute postoperative pain following lower limb joint replacement surgery. The technique is based on systematic infiltration of a mixture of ropivacaine, ketorolac, and adrenaline (RKA) around all structures subject to surgical trauma. The technique, known as local infiltration analgesia (LIA), was developed specifically to avoid sedation and facilitate rapid physiological recovery after lower limb arthroplasty in order to enable early mobilization and discharge ${ }^{[8]}$.

Postoperative pain is usually severe after knee arthroplasty, In the last few years, several studies supporting the benefits of the LIA technique or the modified LIA technique in knee and hip arthroplasties have been published ${ }^{[9]}$.

\section{Visual Analog Scale (VAS)}

The pain VAS is a uni-dimensional measure of pain intensity, which has been widely used in diverse adult populations, including those with musckelo-skeletal diseases ${ }^{[10]}$.

The scale consists of one horizontal or vertical line, usually 10 centimeters in length, that is anchored with verbal descriptors of "no pain "and "pain as bad as it could be". Equipment needed. Pencil and paper. The VAS is also available as a plastic slide ruler and on colored cards that can be given to the respondent ${ }^{[11]}$.

The pain VAS is self-completed by the respondent. The respondent is asked to place a line perpendicular to the VAS line at the point that represents their pain intensity ${ }^{[2,8,9]}$.

A higher score indicates greater pain intensity, based on the distribution of pain VAS scores in postsurgical patients, who described their postoperative pain intensity as none, mild, moderate, or severe, the following cut points on the pain VAS have been recommended; no pain $(0-4 \mathrm{~mm})$, mild pain (5$44 \mathrm{~mm})$, moderate pain $(45-74 \mathrm{~mm})$, and severe pain $(75-100$ $\mathrm{mm})$, The VAS takes one minute to complete ${ }^{[10]}$.

Aim of study: To describe the efficacy of local infiltrative analgesia in TKA patients for decrease the pain score and increase range of motion in early post-operative rehabilitation.

\section{Patients and Method \\ Study design and setting}

A comparative prospective study carried in the period from October 2016 to September 2018 in Medical City/ Hospital of Specialized Surgeries and Private Nursing Home Hospital. (36) Patients enrolled in the current study. Total knee arthroplasty done by the same surgical team and then followed-up to November 2018, (11) of them were males and (25) were females (mean age, 64, 13 years), The objective is to evaluate the local infiltrative analgesia efficacy.

Inclusion criteria: All patients those indicate for primary TKA.

\section{Exclusion criteria}

1. Patients with a history of deep venous thrombosis or pulmonary embolism or on long-term warfarin therapy.

2. Patients undergoing revision procedure.

3. History of allergy or intolerance to 1 of the study drugs.

4. Patient with rheumatoid arthritis.

5. Abnormal liver enzymes.

\section{Operative technique}

Surgery done under general anesthesia or spinal anesthesia according to the senior anesthetist opinion and patient condition and comorbidities. Intra-operative mid-thigh pneumatic tourniquet used with pressure setting (300-350 mm $\mathrm{Hg}$ ) and assistive leg holder had been used for all the patients. 
Intravenous antibiotics infused half an hour before induction of anesthesia and continued for further two days. Then $1 \mathrm{gm}$ of tranxamic acid given i.v before starting the procedure.

Anti-Thrombotic given 12 hours after surgery and continued for further 21 days in the form of subcutaneous low molecular weight heparin 4000 International units. The same surgical team performed the whole surgeries that eliminate technical differences and selection bias. In this study same implant design for all patient which was posterior-cruciate substitution. In this study the approach was for all patients, a Midline skin incision in slight knee flexion extended proximally about $(3 \mathrm{~cm})$ above the upper pole of patella and down to tibial tuberosity and then the arthrotomy done through medial Para patellar approach. We used the femur first technique so we start femur first by setting the IM (intramedullary) angle according to the x-ray measurement and cutting $9 \mathrm{~mm}$ from the more distal condyle then start sizing and chamfering the distal femur.

At this stage, not cut the notch and then shift to finish the tibia bone cut. We used intramedullary guide for tibia cut. The entry point near the base of the anterior tibial spine (anterior $1 / 3$ of the tibial articular surface), reaming the entry hole, before resection confirm the amount of resection by using of stylus $(2 \mathrm{~mm})$ from the affected side and insure proper rotational alignment of the component After cutting the tibia 7-degree posterior slope and perpendicular to the mechanical axis, we used tibial tuberosity as marker to obtain proper rotational alignment of tibial component (medial 1/3). After finishing the extension gap, we start to cut the flexion gap, Used posterior referencing in sizing of femur implant. To obtain rotational alignment of the femoral component we chose 30external rotations. Then start anterior-posterior cut and then chamfering the distal femur. Then started soft tissue balancing, before started to any soft tissue release, removed the osteophyte then carefully examine the flexion gap and extension gap by using spacer block and laminar spreaders, if obtain target (rectangular flexion and extension gap), so continued the procedure. In the LIA group a mixture of 150 $\mathrm{mg}(5 \mathrm{mg} / \mathrm{ml})$ of Bupivacaine and $30 \mathrm{mg}(1 \mathrm{ml})$ of Ketorolac and $0.5 \mathrm{mg}$ epinephrine $(0.5 \mathrm{ml})$, these were mixed with sterile normal saline solution to make up a combined volume of $150 \mathrm{~mL}$ in the operating room.

Fifty $\mathrm{ml}$ of this solution where injected in the subcutaneous tissue in the line of skin incision prior to start the surgery together with infiltration of arthrotomy site before doing the arthrotomy, another fifty $\mathrm{ml}$ of the solution was injected in flexed position into the posterior part of the capsule, the intercondylar area, and around the collateral in ligaments just before cementing the implants by using $50 \mathrm{ml} 23-\mathrm{G}$ needle. Special care was taken to avoid infiltration of the common peroneal nerve and popliteal fossa to avoid injury to vessels and sciatic nerve. The remainder was injected throughout the various soft tissue layers prior to wound closure, in this way, all tissues that were traumatized received the analgesic solution. Then intra articular (deep) drain was placed in the lateral gutter of the knee joint, another (superficial) drain was placed in the subcutaneous tissue. The wound was then closed in layers and adhesive dressings applied, Compression bandages were then applied finally. Postoperatively, regular 1 gm of paracetamol were given three times daily to both groups together with celecoxib $200 \mathrm{mg}$ orally was given twice daily unless contraindicated. First assessment of pain score was four hours after surgery, then 12 hours after surgery, then once daily until the discharge. The assessment of pain according the VAS score was done during the rest and movement. The range of motion measured once daily until discharge and the last measure done six weeks after the surgery by advise the patient to sit on the edge of the bed, the straight leg raising test done at time of discharge, the straight leg raising test was successful if the participant could hold the lower limb $10 \mathrm{~cm}$ from the bed with fully extended knee for 10 seconds. The time to fulfillment of discharge criteria was recorded by a physician who was unaware of the group randomization. The discharge criteria were: mild pain (VAS < 3 at rest) sufficiently controlled by oral analgesics, ability to walk with elbow crutches, ability to eat and drink, and no evidence of any surgical complications. Both groups of patients are involved in same rehabilitation protocol; involving range of motion exercise by using continuous passive motion machine in day one postoperative, then the patient can sit in the bed, get out of bed, and start to walk for many steps by using standard walker, then continue the rehabilitation program.

\section{Statistical analysis}

The data analyzed using Statistical Package for Social Sciences (SPSS) version 25.Categorical data presented by frequencies and percentages. Independent t-test (two tailed) was used to compare the continuous variables among study groups accordingly. Paired t-test was used to compare means of ROM scores pre and postoperatively. Pearson's Chi-square test was used to assess statistical association between study group and straight leg raise test. A level of $\mathrm{P}$-value less than 0.05 was considered significant.

\section{Results}

As shown in table (1), statistically insignificant differences were seen in means of ROM score between study groups preoperatively, one day, two days, and six weeks after operation $(\mathrm{P}=0.141,0.329,0.80$ respectively $)$. While statistically significant difference was seen in means of this score on discharge from hospital $(\mathrm{P}=0.001)$.

Table 1: Comparison in means of ROM score preoperatively, one day, two days, after discharge, and six weeks postoperatively between study groups

\begin{tabular}{|c|c|c|c|}
\hline \multirow{2}{*}{ Variable } & \multicolumn{2}{|c|}{ ROM Score } & \multirow{2}{*}{ P Value } \\
\cline { 2 - 3 } & Group A Mean \pm SD & Group B Mean \pm SD & \\
\hline Preoperatively & $95.75 \pm 27.57$ & $105.75 \pm 10.03$ & 0.141 \\
\hline One Day Postoperatively & $89.37 \pm 11.89$ & $88.50 \pm 7.27$ & 0.329 \\
\hline Two Days Postoperatively & $87.18 \pm 6.04$ & $82.75 \pm 8.18$ & 0.080 \\
\hline On Discharge & $92.50 \pm 6.04$ & $78.75 \pm 6.25$ & 0.001 \\
\hline Six Weeks Postoperatively & $107.81 \pm 7.29$ & $105 \pm 5.61$ & 0.215 \\
\hline
\end{tabular}

\section{Association between LIA and straight leg raise after operation}

Table (2) shows the association between LIA injection and ability to performing straight leg raises after knee arthroplasty. It was clear that there was no statistically significant association between administration of LIA and ability for raising leg straightly after operation $(\mathrm{P}>0.069)$. 
Table 2: Association between LIA and straight leg raises following knee arthroplasty

\begin{tabular}{|c|c|c|c|c|}
\hline \multirow{2}{*}{ Variable } & \multicolumn{2}{|c|}{ Study Groups Total (\%) $\mathbf{n = 3 6}$} & \multirow{2}{*}{ Total } & \multirow{2}{*}{ P- value } \\
\cline { 2 - 3 } & Group A (\%) n= 16 & Group B (\%) n= 20 & \\
\hline \multicolumn{3}{|c|}{ Straight leg raise } \\
\hline Yes & $12(57.9)$ & $9(42.1)$ & $21(58.3)$ & \multirow{2}{*}{0.069} \\
\hline No & $4(26.7)$ & $11(73.3)$ & $15(41.7)$ & \\
\hline
\end{tabular}

Comparison in means of VAS score between study groups four hours, 12 hours, one day, and two days, following total knee arthroplasty

During rest, we found that means of this score four hours, 12 hours, one day, and two days after operation were significantly decreased in patients managed with LIA (group A) compared to means of patients in group B. (2.70 versus $1.87, \mathrm{P}=0.016 ; 3.45$ versus $2.25, \mathrm{P}=0.003 ; 3.90$ versus 3.12 ,
$\mathrm{P}=0.026$; and 4.50 versus $3.50, \mathrm{P}=0.002$ respectively). During movement, statistically insignificant differences in means of VAS score between study groups four hours, 12 hours, and two days after operation $(\mathrm{P}=0.258,0.057$, and 0.284 respectively). While mean of this score one day postoperatively was decreased in patients of group A compared to that in patients of group B and this difference in means was statistically significant $(\mathrm{P}=0.016)$.

Table 3: Comparison in means of VAS score between study groups four hours, 12 hours, one day, and two days, after operation

\begin{tabular}{|c|c|c|c|}
\hline \multirow{2}{*}{ Variable } & \multicolumn{2}{|c|}{ VAS Score } & P \\
\cline { 2 - 4 } & Group A Mean \pm SD & Group B Mean \pm SD value \\
\hline During Rest 4 Hours Postoperatively & $1.87 \pm 0.95$ & $2.70 \pm 0.97$ & 0.016 \\
\hline 12 Hours Postoperatively & $2.25 \pm 1.23$ & $3.45 \pm 1.05$ & 0.003 \\
\hline One Day Postoperatively & $3.12 \pm 1.2$ & $3.90 \pm 0.78$ & 0.026 \\
\hline Two Days Postoperatively & $3.5 \pm 1.09$ & $4.50 \pm 0.68$ & 0.002 \\
\hline During Movement 4 Hours Postoperatively & $2.75 \pm 1.48$ & $3.20 \pm 0.83$ & 0.258 \\
\hline 12 Hours Postoperatively & $3.31 \pm 1.30$ & $4.05 \pm 0.94$ & 0.057 \\
\hline One Day Postoperatively & $3.81 \pm 1.16$ & $4.65 \pm 0.81$ & 0.016 \\
\hline Two Days Postoperatively & $4.65 \pm 1.20$ & $4.95 \pm 0.75$ & 0.284 \\
\hline
\end{tabular}

\section{Discussion}

In group A and group B in the current study, at one and two days postoperatively, means of this score were significantly decreased than pre-operatively $(P<0.05)$. Significant difference was seen in means on discharge from hospital $(\mathrm{P}=0.001)$. Also, no significant association between LIA and ability for raising leg straightly after operation observed $(P$ > 0.069). In Moghtadaei et al. study in 2014, mean of ROM score at the time of discharge was improved in comparison to three months after surgery $\left(69.5^{\circ}\right.$ to $\left.114.4^{\circ}\right)$, in which no significant association found $(P>0.05)^{[11]}$.

Also in Carli et al. study in 2010, maximal knee extension improved in group of patients managed with LIA (Group I) and other one managed with nerve block (Group F) at six weeks with no difference between them. Maximal knee flexion decreased significantly in Group I $(-7.8 \%)$ but not significantly in Group $F(-6.0 \%)$, with no differences between groups regarding absolute values ${ }^{[12]}$.

In Chaumeron et al. study in 2013 on patients managed with ILA and other with nerve block, the pattern of postoperative knee-assisted flexion between groups significantly differed over days one through five $(\mathrm{p}=0.020)$. Also there was a significant greater knee assisted flexion on the day of surgery in the LIA group $(p=0.001)$ but none at other follow-ups $(P>0.05)$. The pattern of free knee flexion between groups differed significantly over days one through five $(\mathrm{p}=0.001)$. Furthermore, the LAI group had increased free knee flexion on the day of surgery $(\mathrm{p}=0.042)$ but none at other follow-ups $(P>0.05)^{[13]}$.

Mullaji et al. in 2010, conducted a study of bilateral TKAs in which one knee received LIA and the other knee did not. Patients had greater active flexion up to four weeks and superior quadriceps recovery up to two weeks in the LIA in knee ${ }^{[14]}$.

Relief of acute pain after TKA represents a major therapeutic challenge as post-operative pain hinders early mobilization and rehabilitation with subsequent consequences on mobility, duration of hospitalization and overall recovery. It offers the benefits of blocking pain influx at its origin and maximizing muscle control ${ }^{[22]}$. The new rehabilitation protocols emphasizing ROM restoration in out clinic form (outpatients and patients outside the clinic) which bases the discharge criteria on the physical ability not pain control. Furthermore, good perioperative analgesia facilitates rehabilitation, improves patient satisfaction ${ }^{[15]}$.

Visual analogue scale (VAS) score results among study groups shows that the means of this score during rest were significantly decreased in group A than in group B in all periods. There is significantly decreased in one day in-group A compared to $\mathrm{B}(\mathrm{P}=0.016)$.

Andersen et al. study in 2010, found that VAS at rest and during mobilization were statistically significantly lower in patients received LIA than those not received it during the whole study period, with the exception of pain scores during mobilization at $24-48 \mathrm{~h}$ after surgery $(\mathrm{p}=0.05)^{[16]}$.

Busch and his colleagues in a study at 2006, found that patients who had received the LIA used significantly less patient-controlled analgesia at six, at twelve, and over the 1sttwenty-four hours after the surgery. In addition, they had higher VAS scale for patient satisfaction and lower VAS scale for pain during activity in the post-anesthetic-care unit and four hours after the operation, concluded that intraoperative LIA with multimodal drugs can significantly reduce the requirements for analgesia and improve patient satisfaction, with no apparent risks, following TKA ${ }^{[17]}$.

Furthermore, Vendittoli et al. study in 2006 stated that multimodal perioperative analgesia protocol that included LIA, offered improved pain control and minimal side effects to patients undergoing TKA. It also confirmed the safety of the protocol ${ }^{[18]}$.

In another study, Toftdahl et al. study in 2007, found that peri and intra-articular application of analgesics by infiltration and bolus injections can improve early analgesia and mobilization for patients undergoing TKA, when noticed that patients with 
LIA could significantly walk $<3$ meters on the 1stpostoperative day $(p<0.001)$. Same patients had significantly lower pain scores during activity and lower consumption of opioids on the 1stpostoperative day ${ }^{[19]}$

In Albrecht et al. study in 2016, a reduction in analgesia consumption at 12 hours postoperatively observed and the difference between groups reached statistical significance $(P<0.0001)$. Also they found no significant differences in pain scores at rest $(\mathrm{P}=0.80)$ or pain scores on movement $(\mathrm{P}=0.64)$ on postoperative day one. Similarly, length of stay was statistically different but without direct clinical relevance. They concluded that LIA provides similar postoperative analgesic efficacy to nerve block after TKA, but requires a higher dose of local anaesthetic and reported incidence of complications does not differ between groups ${ }^{[20]}$.

In comparison to other studies, Andersen and colleagues found in their study in 2010, that mean of age of the patients was 67 years (in range of 63-72), a male predominance observed (57\%) with Male: Female ratio was 1.3:1 and the mean of BMI level was $28 \mathrm{Kg} / \mathrm{m} 2$ (in range of $25-32 \mathrm{Kg} / \mathrm{m} 2$ ). The median length of hospital stay was 4 days (Between3-5) days in patients received LIA; however, discharge criteria were significantly earlier in same group 3 days (between 33.5) days) than those not received LIA 4 days (between 3-5 days) $(p<0.004)^{[15]}$.

Moghtadaei et al. study in 2014, observed the mean and SD of the patients age was $64 \pm 6.9$ years, and the mean and SD of the BMI was $27 \pm 2.5 \mathrm{Kg} / \mathrm{m} 2$, with male represented the highest proportion, when constituted $72 \%$ of them, with male: female ratio was 2.6:1. Concerning number of hospitalization days in those managed with LIA were 5.8 days and those without LIA, they were 5.3 days on average with no significant differences between both groups ${ }^{[11]}$. Finally, Yoon et al study in 2010, found that mean of the participant's age was 70 years (ranged from 34-83 years) and a female represented the vast majority of their study, when constituted $94.1 \%$ of them, with female to male ratio was $16: 1$, also mean of BMI was $26.4 \mathrm{Kg} / \mathrm{m} 2$ (ranged from $19.1-34.2 \mathrm{Kg} / \mathrm{m} 2)^{[21]}$.

\section{Conclusion}

LIA is a simple effective method to reduce pain and improve physiotherapy outcome also reduces hospital stay in patients undergoing TKA.

\section{Recommendations}

1. A larger sample size and longer period of study to decrease the chance of error and bias.

2. Long period of follow-up to assess the effectiveness of LIA on long-term functional outcome.

3. Prepare and mix the content of LIA by one of surgical team in the field of surgical sets to ensure complete aseptic technique.

4. Availability of drugs and anesthetist are mandatory for continuous using this method.

No conflicts of interest

Source of funding: self

Ethical clearance: was taken from the scientific committee of the Iraqi Ministry of health

\section{References}

1. Al-Sharqi SA, Wahab MS, Hussainy SK. Histopathological study in posterior cruciate ligament of osteoarthritis and rheumatoid arthritis in Iraqi patients. American journal of medicine and medical sciences
2013;3(1):10-6.

2. Richard Snell S. Clinical Anatomy by Regions 2012,500.

3. O'Donnell R, Dolan J. Anaesthesia and analgesia for knee joint arthroplasty. Bja Education 2018;18(1):8-15.

4. Newman Pydisetty R, Ackroyd C. Unicompartmental or total knee replacement the 15-year results of a prospective randomised controlled trial. Journal of Bone \& Joint Surgery, British 2009;91:52-57.

5. $\mathrm{Hu} \mathrm{B}, \mathrm{Lin} \mathrm{T}$, Yan SG, Tong SL, Yu JH, Xu JJ, Ying YM. Local infiltration analgesia versus regional blockade for postoperative analgesia in total knee arthroplasty: a metaanalysis of randomized controlled trials. Pain Physician 2016;19(4):205-14.

6. Kurosaka K, Tsukada S, Seino D, Morooka T, Nakayama $\mathrm{H}$, Yoshiya S. Local infiltration analgesia versus continuous femoral nerve block in pain relief after total knee arthroplasty: a randomized controlled trial. The Journal of arthroplasty 2016;31(4):913-7.

7. Dalury DF. A state-of-the-art pain protocol for total knee replacement. Arthroplasty today 2016;2(1):23-5.

8. Kerr DR, Kohan L. Local infiltration analgesia: a technique for the control of acute postoperative pain following knee and hip surgery: a case study of 325 patients. Acta orthopaedica 2008;79(2):174-83.

9. Essving P, Axelsson K, Kjellberg J, Wallgren Ö, Gupta A, Lundin A. Reduced morphine consumption and pain intensity with local infiltration analgesia (LIA) following total knee arthroplasty: a randomized double-blind study involving 48 patients. Acta orthopaedica 2010;81(3):35460.

10. Hawker GA, Mian S, Kendzerska T, French M. Measures of adult pain: Visual analog scale for pain (vas pain), numeric rating scale for pain (nrs pain), mcgill pain questionnaire (mpq), short-form mcgill pain questionnaire (sf-mpq), chronic pain grade scale (cpgs), short form-36 bodily pain scale ( $\mathrm{sf}-36 \mathrm{bps}$ ), and measure of intermittent and constant osteoarthritis pain (icoap). Arthritis care \& research 2011;63(S11):S240-S252.

11. Moghtadaei M, Farahini H, Faiz SH, Mokarami F, Safari S. Pain management for total knee arthroplasty: singleinjection femoral nerve block versus local infiltration analgesia. Iranian Red Crescent Medical Journal 2014;16(1):e13247.

12. Carli F, Clemente A, Asenjo JF, Kim DJ, Mistraletti G, Gomarasca $\mathrm{M}$ et al. Analgesia and functional outcome after total knee arthroplasty: periarticular infiltration vs continuous femoral nerve block. British Journal of Anaesthesia 2010;105(2):185- 95.

13. Chaumeron A, Audy D, Drolet P, Lavigne M, Vendittoli PA. Periarticular injection in knee arthroplasty improves quadriceps function. Clinical Orthopaedics and Related Research®. 2013;471(7):2284-95.

14. Mullaji A, Kanna R, Shetty GM, Chavda V, Singh DP. Efficacy of periarticular injection of bupivacaine, fentanyl, and methylprednisolone in total knee arthroplasty: A prospective, randomized trial. J Arthroplasty 2010;25:851-857.

15. Salinas FV, Liu SS, Mulroy MF. The effect of singleinjection femoral nerve block versus continuous femoral nerve block after total knee arthroplasty on hospital length of stay and long-term functional recovery within an established clinical pathway. Anesth Analg 2006;102(4):1234-9.

16. Andersen KV, Bak M, Christensen BV, Harazuk J, Pedersen NA, Søballe K. A randomized, controlled trial 
comparing local infiltration analgesia with epidural infusion for total knee arthroplasty. Acta orthopaedica 2010;81(5):606-10.

17. Busch CA, Shore BJ, Bhandari R, Ganapathy S, MacDonald SJ, Bourne RB et al. Efficacy of periarticular multimodal drug injection in total knee arthroplasty: a randomized trial. JBJS 2006;88(5):959-63.

18. Vendittoli PA, Makinen P, Drolet P, Lavigne M, Fallaha $\mathrm{M}$, Guertin MC et al. A multimodal analgesia protocol for total knee arthroplasty: a randomized, controlled study. JBJS 2006;88(2):282-9.

19. Toftdahl K, Nikolajsen L, Haraldsted V, Madsen F, Tønnesen EK, Søballe K. Comparison of peri-and intraarticular analgesia with femoral nerve block after total knee arthroplasty: a randomized clinical trial. Acta orthopaedica 2007;78(2):172-9.

20. Albrecht E, Guyen O, Jacot-Guillarmod A, Kirkham KR. The analgesic efficacy of local infiltration analgesia vs femoral nerve block after total knee arthroplasty: a systematic review and meta-analysis. BJA: British Journal of Anaesthesia 2016;116(5):597-609.

21. Yoon HS, Han CD, Yang IH. Comparison of simultaneous bilateral and staged bilateral total knee arthroplasty in terms of perioperative complications. The Journal of arthroplasty 2010;25(2):179-85. 